simple proof of the theorem that a skew symmetric determinant of even order is a perfect square. This can be done by examination of the way in which cycles of two indices may be agglomerated in cycles of more indices, but it would hardly seem to be so simple as Sylvester believed. One can, however, easily enumerate the terms in the square root, the Pfaffian. For the squared terms in the skew determinant correspond exclusively to permutations containing cycles of two indices only, since $(i j)$ connotes $-a_{i j} a_{j i}$, or $a_{i j}^{2}$. Thus we have to find in how many ways $2 m$ indices may be put into $m$ such cycles. For first cycle take 1 and any $a$ from the remaining $2 m-1$ indices; for second cycle take the next surviving index in natural order and any $b$ from the remaining $2 m-3$; and so proceed. The number of terms in the Pfaffian is thus $(2 m-1)(2 m-3)(2 m-5) \ldots$ 5.3.1, a factorial composed of odd numbers. This is a well-known result.

The Mathematical Institute, 16 Chambers Street, Edinburgh.

\title{
On the Newton-Raphson method of Approximation
}

By H. W. Richmond, F.R.S.

1. The Method.-An equation $F(x)=O$ has a root $x=r$, not known exactly. From a first approximation to $r, x=a$, a second approximation, $x=b$, is obtained from the formula

$$
b=a-F(a) / F^{\prime}(a)
$$

From $b$ a third approximation, $x=c$, is obtained by the same formula, and so on. The method is pointless unless the successive

Recently I have had occasion to read the accounts of this method given in various books; among them

(a) Whittaker \& Robinson, Calculus of Observations.

(b) Weber, Algebra.

(c) Todhunter, Theory of Equations.

The last, a forgotiten text-book of 1880 , contains the fullest account of the method known to me. I venture to offer some comments and criticisms. 
approximations do actually tend to $r$; a rule that ensures this is due to Fourier. ${ }^{1}$

The first point that I wish to emphasise is that the method from beginning to end rests upon geometrical conceptions. The graphs in $(a)$ and $(c)$ of a normal function $F(x)$ show far better than Fourier's memoir the inwardness of the formula and the method. They justify Fourier's rule as to the side from which $r$ must be approached. They indicate the infrequent circumstances under which the Newton-Raphson method cannot be applied.

They do more. They compel attention to a fact commonly ignored, the extraordinarily rapid approach of the later approximations to the true value of $r$. For in the diagrams $(a)$ and $(c)$, if the construction is carried a stage further, the points obtained are so closely packed together that the figure becomes microscopic; distances, i.e. numerical differences, are inappreciable in the scale previously adopted.

Test this by the simplest irrational number, $\sqrt{ } 2$. The continued fraction furnishes a series of convergents

$$
1,3 / 2,7 / 5,17 / 12,41 / 29,99 / 70,239 / 169,577 / 408, \ldots
$$

each known to be nearer to $\sqrt{ } 2$ than any fraction with a smaller denominator. The Newton-Raphson method, applied when $F(x)$ is $x^{2}-2$, gives

$$
3 / 2,17 / 12,577 / 408,665857 / 470832, \ldots
$$

as approximations, so that in two steps we reach the eighth, in three the sixteenth convergent, the last a fraction equal to $\sqrt{ } 2$ with a small error in the twelfth decimal place.

Formula (i) provides an algebraic explanation. Write $r+a$ and $r+\beta$ in place of $a$ and $b$ so that $a$ and $\beta$ are the errors in the two approximations. Expand $F(r+a)$ and $F^{\prime}(r+a)$ in powers of a. The expansion of $\beta$ in powers of $a$ takes the form

$$
\beta=g a^{2}+h a^{3}+k a^{4}+\ldots
$$

$g$ being in fact $\frac{1}{2} F^{\prime \prime}(r) / F^{\prime}(r)$. The errors in the second, third, fourth, ... approximations are therefore of the orders

1 Oeuvres, Vol. 2, pp. 243-253. 
$a^{2}, a^{4}, a^{8}, a^{16} \ldots$, their leading terms being $g a^{2}, g^{3} a^{4}, g^{7} a^{8}, g^{15} a^{16}, \ldots$ If $a$ and $g a$ are small, the errors in the successive approximations rapidly become infinitesimal.

If it is asked whether this result will often be of use to the practical computer, the answer must be that it is doubtful. He probably wishes to determine one or two more figures of some number; a method which will, maybe, double the number of known significant figures is too sweeping. The equation he is solving is possibly not a clean cut algebraical equation such as is suggested by our notation $F(x)=0$. Also the approximations are presented as fractions with a large numerator and denominator, which is inconvenient even in these days of computing machines.

The rule given by Fourier may be stated in several ways. As the variable $x$ passes from $r$ the unknown root to $a$ the first approximation, $F(x)$ and $F^{\prime \prime}(x)$ must be of the same sign. In the graph of $F(x)$ the curve must be convex to the foot of the ordinate. As $x$ passes from $r$ to $a$ the inclination (without regard to sign) of the tangent to the $x$-axis must increase; i.e., $\left|F^{\prime}(x)\right|$, or the square of $F^{\prime}(x)$, must increase. Yet many books commit themselves to the statement that $F^{\prime}(x)$ and $F^{\prime \prime}(x)$ must not vanish for values of $x$ between $r$ and $a$. $\quad F^{\prime}(x)$ cannot vanish unless Fourier's rule is violated.

Another such statement is that, if $F(x)$ has two roots close to one another, the Newton-Raphson method becomes untrustworthy. If Fourier's rule is followed this statement is simply untrue. Even if Fourier's rule is ignored, the statement is open to criticism. If $F^{\prime}(a)$ is 0 , the value of $b$ is infinite : that a second approximation should be infinite is manifestly absurd. If $F^{\prime}(a)$ is small the second approximation $b$ will differ widely from $a$; $r$ will lie between $a$ and $b$ (as is usual when Fourier is ignored) and $b$ will very possibly be much further from $r$ than $a$ is. Whether $F(x)$ has a second root close to $r$ or no is immaterial. If $F^{\prime}(a)$ happens to be small and Fourier's rule is violated, trouble may be expected.

Formula (1) may be replaced by another in which $\beta$ (the error in $b$ the second approximation) is of order $a^{3}$, viz.,

$$
b=a-F F^{\prime} \div\left(F^{2}-\frac{1}{2} F F^{\prime \prime}\right)
$$

$F, F^{\prime}, F^{\prime \prime}$ being functions of the variable $a$. It is unlikely that this 
formula will be of use; but it assumes a simple form in the approximation to the cube root of a number $R$, viz.

$$
\frac{a-b}{a+b}=\frac{1}{3} \frac{a^{3}-R}{a^{3}+R}
$$

For the $n^{\text {th }}$ root of $R$ there is a similar formula

$$
\frac{a-b}{a+b}=\frac{1}{n} \frac{a^{n}-R}{a^{n}+R}
$$

the order of the error in $b$ again being the cube of that in $a$.

King's Col.lege,

Cambridge.

\title{
Some Parameters of Sampling Distributions Simply Obtained
}

\author{
By L. M. Brown.
}

In the theory of statistics a set of quantities $a_{1}, a_{2}, \ldots, a_{v}$ is considered, and called a distribution. The moments of this distribution about its origin are defined by the equations

$$
\mu_{1}^{\prime}=\frac{1}{\nu} \Sigma a_{i} ; \quad \mu_{2}^{\prime}=\frac{1}{\nu} \Sigma a_{i}^{2} ; \quad \mu_{3}^{\prime}=\frac{1}{\nu} \Sigma a_{i}^{3} .
$$

The Nean $M$ of the distribution is defined as $\mu_{1}^{\prime}$; if $x_{i}=a_{i}-M$, then the moments of the distribution about its mean are defined by the equations

$$
\mu_{1}=0 ; \quad \mu_{2}=\frac{1}{\nu} \Sigma x_{i}^{2} ; \quad \mu_{3}=\frac{1}{\nu} \Sigma x_{i}^{3} .
$$

It is easy to show that $\mu_{2}=\mu_{2}^{\prime}-M^{2}$ and that $\mu_{3}=\mu_{3}^{\prime}-3 M \mu_{2}^{\prime}+2 M^{3}$. The variance, $\sigma^{2}=\mu_{2}$, of the distribution is a measure of its dispersion or spread, and $\beta_{1}=\mu_{3}^{2} / \mu_{2}^{3}$ is a measure of its asymmetry or skewness. All this appears in any elementary account of the subject. 